Sharif University of Technology
Scientia Iranica
SCIENTIA

\title{
Symbiotic organisms search-based multi-objective optimal placement of distributed generators considering source and load uncertainty
}

\author{
K. Chakraborty ${ }^{a}$, G. Deb ${ }^{b, *}$, and S. Sharma ${ }^{c}$ \\ a. Department of Electrical Engineering, Tripura Institute of Technology, Narsingarh, India. \\ b. Department of Electrical Engineering, Tripura University, Suryamaninagar, India. \\ c. Department of Electrical Engineering, National Institute of Technology-Agartala, Jirania, India.
}

Received 9 June 2020; received in revised form 4 May 2021; accepted 19 July 2021

KEYWORDS
Distributed
generation;
Radial distribution
network;
Symbiotic organisms
search;
Voltage security;
Voltage stability
indicators.

\section{Introduction}

Due to the increasing environmental concerns as well as electricity market restructuring, Distributed Generation (DG) has been widely applied to the distribution networks. Integration of DG into the distribution system affects its power flow, voltage profile, voltage stability, reliability, and loadability [1]. Proper allot-

*. Corresponding author. Tel.: 91-381-7963969

E-mail addresses: kabir_jishu@rediffmail.com (K.

Chakraborty); gagarideb@tripurauniv.ac.in (G. Deb);

sharmistha.sharma34@yahoo.com (S. Sharma)

doi: $10.24200 /$ sci. 2021.56149 .4575 ment of DG to distribution networks can improve their operating state [2]. Due to the high $R / X$ ratio of the distribution system, the power loss is greater than that in the transmission system. As the last assembly between the generation site and consumer, the distribution system has one main drawback, i.e., power quality problem. In order to overcome this problem, it is required to install renewable DGs of ideal size in optimal locations in distribution systems [3]. Given that the arbitrary placement of DG may increase the power loss, the power flow may be in the opposite direction, which may increase heat generation in feeders [4].

In the literature, different technical and economic objectives were utilized for DG installation in the distribution networks [5]. In [6], a probabilistic Voltage 
Stability Index (VSI) was used that could combine the cumulants using the maximum entropy algorithm to study the radial distribution network considering the uncertainty. In [7], a new and fast method for tracing the P-V curve was established to analyze the voltage stability. An analytical method of DG assignment and sizing was proposed in [8] to reduce the system loss. Backtracking search optimization algorithm was employed in [9] to obtain the objective function capable of reducing the system real power loss and improving the voltage profile. In [10], many conventional and metaheuristic techniques were discussed followed by reviewing different analytical techniques for DG allocation. In [11], a multi-objective function was introduced to minimize the annual total cost and distribution network risk, considering the correlations between the generation and load uncertainties. Grey wolf optimization technique was implemented in [12] to minimize the reactive power loss and improve the voltage profile. Modified traditional firefly method was also used in [13] for optimal allocation and size determination of the DG with the objective of reducing the active power or daily energy losses. Shuffled bat algorithm proposed in [14] could minimize the power losses, cost, and voltage deviation. Ant Lion Optimization (ALO) technique presented in [15] minimized the line loss and improved the voltage profile as well as VSI for optimal DG placement within the distribution system. Placement of large-scale utility-owned wind DG was achieved in [16] using the cumulative probabilistic distribution function and congestion improvement ratio. In [17], a teaching-learning-based method for DG placement was suggested where the objective function comprised the loss reduction, voltage profile improvement, annual saving improvement, and VSI maximization. Monte Carlo-dependent multi-objective optimal placement of renewable DG using open computing language was done by authors in [18] based on the line loss and cost of DG as the objective function. In [19], the investment cost, highest income, lowest environmental cost, and minimum loss were considered as the objective functions for DG assignment under uncertainty through Dempster-Shafer evidence theory and affine arithmetic method. A new stochastic method for DG allocation in the distribution system with the objective of loss reduction based on the optimal power flow and sensitivity analysis was proposed in [20]. Genetic algorithm was used in [21] to determine the optimal size of DG and optimal reconfiguration of the network, aiming to minimize the line losses as well as total harmonic distortion and to improve the voltage profile of the network. A novel multi-objective opposition-based chaotic differential evolution algorithm was proposed in [22] for ideal placement of DG, considering the reduction of the power loss, yearly economic loss, and voltage deviation as the objective function. In [23], uncertainties in the output power of the renewable energy sources were evaluated by proposing a new formulation obtained from Particle Swarm Optimization (PSO) algorithm-based optimal location and sizing wind, solar, and fuel cells in the distribution system. Multi-objective Taguchi approach was presented in [24] for best allotment of DG in both small and large distribution systems with the objective functions of minimization of the real power loss, minimization of reactive power loss, minimization of node voltage deviation, maximization of Voltage Stability Margin (VSM), and maximization of VSI. A novel chaotic stochastic fractal search method was used in [25] to determine the optimal siting, sizing, and number of DG units in the distribution system where the objective function was power loss minimization. Under different loading conditions, the optimal sizing and siting of DG vary. In [26], examination as to how the optimal solution would change as a result of different load compositions was conducted by proposing a local PSO variant algorithm as the solution algorithm. A hybrid technique for reducing the distribution system losses was utilized in [27] to optimize the position and size of DG units. The hybrid technique is a combination of Grasshopper Optimization and Cuckoo Search algorithms. A novel approach to DG placement was presented in [28] where weak buses were identified first using Voltage Stability Margin Index (VSMI) while the optimum size of DG unit was computed via MATLAB curve-fitting approximation. In [29], a recent optimization method called improved raven roosting optimization algorithm was used for optimal placement of DG in the radial distribution system where the technical issues were included via a weighted multiobjective index. In [30], the Pareto-front of nondominated solutions was obtained from the contradictory relationship between the reduction in the MVA rating of DGs and that in the power losses of the system using multi-objective differential evolution algorithm. In [31], minimization of the system total real power loss was mentioned as the main objective while determining the optimal location and size of different DG types using a hybrid technique composed of Weight-Improved Particle Swarm Optimization (WIPSO) and Gravitational Search Algorithm (GSA) called the hybrid WIPSO-GSA algorithm.

The discussed heuristics optimization methods in the literature succeeded in providing an approximate solution when the classical methods failed to do so; however, the convergence speed of these algorithms is slow, and many of them fail to find the optimal solution. On the contrary, analytical techniques alone are not enough for optimal placement of DGs [32]. In the earlier research works of the same authors, DG placement problem was solved through Spider Monkey Optimization (SMO) technique with only voltage devi- 
ation minimization as the objective function. Of note, a correct solution may not be found through the SMO technique due to the arbitrary parameters [33].

In this regard, in an effort to improve their previous work, the authors of this study used Symbiotic Organisms Search (SOS) and considered a multiobjective optimization problem consisting of simultaneous voltage deviation minimization and VSI maximization. According to the findings, SOS outperformed some other population-based algorithms since it offered mutualism strategy to modify the candidate solutions, a strategy that was not used in PSO and Differential Evolution. A unique SOS characteristic is its use of another mutation operator called Parasitism. The main advantage of SOS is that the specific parameters are not required to run the algorithm [34].

The referenced studies demonstrated the effect of DG allocation on the loss reduction, voltage profile improvement, and indicator value enhancement. However, the main focus of this study was to show the effect of DG allocation on the voltage security state of the distribution system. To increase the efficiency of the SOS, weighted sum technique [35] was employed to solve the multi-objective problem, which could generate different Pareto optimal solutions. Due to the power system restructuring and unpredictable nature of renewable energy sources, it is very important to model the uncertainty of the power systems. The current study utilized a 2m Point Estimation Method (2m PEM) as a non-iterative, efficient, simple, and easy technique with no convergence problem to model the generator and load uncertainties [36].

SOS technique was also used for DG placement. Based on this algorithm, the candidate buses for DG allocation along with DG sizes were calculated. In addition, the outcomes of SOS were crosschecked using Quasi-Oppositional Swine Influenza Model Based Optimization with Quarantine (QOSIMBO-Q) and Swine Influenza Model Based Optimization with Quarantine (SIMBO-Q) [37] to prove its superiority. During contingency, the state of the distribution systems under test was categorized into three operating states namely secure, intermediate, and emergency based on the VSI values [38]. This methodology was tested on two standard IEEE distribution networks, and the obtained results revealed that the SOS technique could ensure the utmost voltage profile improvement and improve the voltage security states of the distribution system since the proposed scheme could increase the indicator value.

\section{Problem formulation}

\subsection{Objective functions}

\subsubsection{Improvement of voltage profile}

Voltage security of a system depends mostly on the bus voltage magnitudes [39]. To be specific, the system with less deviation of the bus voltages from the rated magnitude is more secure. In this regard, in the current study, minimization of the voltage variation was considered as the first objective function for voltage profile improvement in each bus.

The objective function for voltage profile improvement or voltage variation reduction for $N$ node network can be written as:

$$
f_{1}=\operatorname{Min} \sum_{i=1}^{N}\left(\left|V_{i}\right|-\left|V_{\text {rated }}\right|\right)^{2},
$$

where $V_{i}$ is the magnitude of voltage at Node $l$, and $V_{\text {rated }}$ the magnitude of the rated voltage (which is 1 p.u in this study).

\subsubsection{Improvement of VSI}

In case of contingency in the distribution system, the value of the system indicator will vary. For this reason, improvement of the stability indicator is considered as the second objective function. Several indices were developed by the researchers to analyze the operating condition of the power system. The VSI proposed by Chakravorty and Das [38] is given below:

$$
V S I=V_{k}^{4}-4\left[P_{l} X-Q_{l} R\right]^{2}-4\left[P_{l} R+Q_{l} X\right] V_{k}^{2},
$$

where $R$ is the line resistance, $X$ the line reactance, $V_{k}$ the voltage of sending end node, $P_{l}$ the total real power load available at receiving end node, and $Q_{l}$ the total reactive power load available at the receiving end node.

For stable operation of the radial distribution system, the range of VSI should be between 0 and 1 .

The bus for which the value of VSI is the lowest is considered as the weakest bus in the system. In this situation, either Eq. (2) or $f_{2}$ should be maximized which is mathematically represented as follows:

$$
f_{2}=\operatorname{Min}\left(\frac{1}{\operatorname{VSI}(l)}\right)
$$

where $l=1,2,3, \ldots, N$.

The VSI value determines the voltage stability condition of the bus in the distribution system. The DGs should be installed on the bus with the lowest VSI value given the higher possibility of the collapse of the voltage of the mentioned bus. The higher value of the VSI is indicative of the less chance of voltage collapse and in the current research, the VSI improvement was considered the second objective function, i.e., maximizing Eq. (2) or minimizing $f_{2}$. The third objective function is a multi-objective problem consisting of minimization of voltage deviation and maximization of VSI. As in the multi-objective problem, both objective functions should be written in either minimization or maximization forms, and maximization of the VSI part is converted into the minimization of the inverse of VSI. 


\subsubsection{Improvement of voltage profile and VSI}

The third objective function is a multi-objective problem where both voltage profile and VSI should be simultaneously improved, as expressed in the following equation:

$$
f_{3}=\operatorname{Min}\left(f_{1} \times w+f_{2} \times(1-w)\right),
$$

where $f_{1}$ and $f_{2}$ are the functions of the voltage profile and VSI improvements, respectively, and $w$ is the weighting factor which varies uniformly between 0 and 1 with a step of 0.05 .

\subsection{Constraints}

\subsubsection{Equality constraint}

The equality constraint here is the power balance equation that should be satisfied at each bus:

$$
\begin{aligned}
& P_{g l}=P_{d l}+V_{l} \sum_{k=1}^{N} V_{k} Y_{l k} \cos \left(\delta_{l}-\delta_{k}-\theta_{l k}\right), \\
& Q_{g l}=Q_{d l}+V_{l} \sum_{k=1}^{N} V_{k} Y_{l k} \sin \left(\delta_{l}-\delta_{k}-\theta_{l k}\right),
\end{aligned}
$$

where $P_{g l}$ and $Q_{g l}$ are the generated active and reactive power at bus 1, respectively, $P_{d l}$ and $Q_{d l}$ are demands for active and reactive power at bus 1, respectively, $V_{k} \angle \delta_{k}$ and $V_{l} \angle \delta_{l}$ are the voltages of the $k$ th and $l$ th buses, respectively, $Y_{l k}$ is the magnitude of the $l k$ th element of admittance matrix, and $N$ is the total number of buses.

\subsubsection{Inequality constraints}

In this optimization problem, there are three inequality constraints that are discussed in the following subsections.

- Limits of bus voltage magnitude. The voltage magnitude should lie between the upper and lower values at every node of the network. Voltage magnitude constraint can be written as:

$$
V_{l}^{\min } \leq V_{l} \leq V_{l}^{\max }
$$

where $V_{l}^{\text {min }}$ and $V_{l}^{\text {max }}$ are the minimum and maximum bus voltage magnitudes equal to $0.95 \mathrm{p} . \mathrm{u}$ and 1.05 p.u, respectively.

- DG active power constraints. DG capacity at any given location is dependent on the sources of energy available at that site. In this respect, it is important to keep the DG active power capacity within the upper and lower limits [40].

The range of $\mathrm{DG}$ active power is determined in the following:

$$
P_{g l}^{\min } \leq P_{g l} \leq P_{g l}^{\max } .
$$

Here,

$$
\sum P_{g l} \leq \sum_{i=2}^{N} P_{L O A D}
$$

where $P_{g l}^{\min }$ and $P_{g l}^{\max }$ are the minimum and maximum values of the total real power production of DG, $P_{L O A D}$ is the total real power load linked in the network, and $N$ represents the number of nodes. In this study, the power injected by the three DGs is considered as less than or equal to the total real power load of the system.

- Line capacity constraint. The amount of complex power flowing through a line should be less than its rated value, which is expressed by the following equation:

$$
S_{l} \leq S_{(\text {rated })},
$$

where $S_{l}$ is the actual complex power at bus 1, and $S_{(\text {rated })}$ the rated complex power at bus 1 .

\section{Modeling of uncertainties}

The output power of the solar energy-based DG is not constant given its dependency on the solar intensity, which is also variable. The output power obtained from the wind power-based DG is dependent on the wind speed that also varies from time to time. The amount of load demand also changes. Normally, deterministic forecasts give information about the historical performance of the proposed technique, but they are unable to estimate the uncertainty associated with the given prediction. In this situation, uncertainty is expressed in the form of probabilistic forecasts. Use of probabilistic forecasts can bring about more economic benefits [41]. This research introduced some variable parameters such as solar intensity, wind speed, and load demand based on the Probability Density Function (PDF).

The significant superiority of the probabilistic approach over its deterministic counterpart is that the potential scenarios of the stratigraphic configuration and stratum properties can be sampled according to the characterized uncertainty, and this type of sampled scenarios is more systematic and complete than the other types in the limited scenarios given by deterministic approach [42].

\subsection{Uncertainty of wind power}

Since wind speed is stochastic in nature, the power output from wind turbine is uncertain. In this paper, the PDF of wind is described based on Weibull distribution and expressed as follows [11]:

$$
f(v)=\left(\frac{k}{c}\right)\left(\frac{v}{c}\right)^{(k-1)} \exp \left[-\left(\frac{v}{c}\right)^{k}\right],
$$

where the constants of Weibull distribution are $c$ and $k$.

Normally, the wind speed is monitored at a height of $10 \mathrm{~m}$ and is converted into the wind speed at required height based on the following equation [11]: 


$$
V_{H}=V_{10}\left(\frac{H}{10}\right)^{\frac{1}{7}}
$$

where $V_{H}$ is the wind speed at height $H$ and $V_{10}$ the wind speed at $10 \mathrm{~m}$. Based on the parameters of wind turbine generator and $V_{H}$, the value of $P_{w t g}$ can be calculated through the following equation [11]:

$$
P_{w t g}= \begin{cases}0 & 0 \leq V_{H} \leq V_{c i} \text { or } \quad V_{c o} \leq V_{H} \\ P_{w t g}^{r} \frac{V_{H}-V_{c i}}{V_{r}-V_{c i}} & V_{c i} \leq V_{H} \leq V_{r} \\ P_{w t g}^{r} & V_{r} \leq V_{H} \leq V_{c o}\end{cases}
$$

where $P_{w t g}^{r}$ is the rated active power of wind turbine generator, and $V_{c i}, V_{r}, V_{c o}$ are cut in speed, rated speed, and cut out speed of wind turbine generator, respectively.

\subsection{Uncertainty of solar power}

Although many factors can affect the output power of the solar photo voltaic cell, the solar intensity, $S$ in particular, is emphasized here for simplicity. The stochastic light intensity, which is also known as Beta distribution, can be written as follows [11]:

$$
f(s)=\frac{\Upsilon(\alpha+\beta)}{\Upsilon(\alpha) \Upsilon(\beta)}\left(\frac{S}{S_{r}}\right)^{\alpha-1}\left(1-\frac{S}{S_{r}}\right)^{\beta-1},
$$

where $\alpha$ and $\beta$ are known as the shape parameters of Beta distribution; $S_{r}$ and $\tau($.$) are the rated light$ intensity and Gamma function, respectively.

The active power obtained from the solar photo voltaic cell can be calculated from the following equation [11]:

$$
P_{p v g}= \begin{cases}P_{p v g, r} \frac{S}{S_{r}}, & S \leq S_{r} \\ P_{p v g, r}, & S>S_{r}\end{cases}
$$

where $P_{p v g, r}$ is the rated active power of the solar photo voltaic cell.

\subsection{Uncertainty of load}

The load demand of the consumers varies on different days, hence considered a stochastic variable. To better describe the load uncertainty, normal distribution was taken into consideration in this study. The PDF for the load demand can be written as [11]:

$$
\begin{aligned}
& f\left(P_{l}\right)=\frac{1}{\sigma_{p} \sqrt{2 \Pi}} \exp \left[-\frac{\left(P_{l}-\mu_{p}\right)^{2}}{2 \sigma_{p}^{2}}\right], \\
& Q_{l}=P_{l} \tan \left(\varphi_{l}\right),
\end{aligned}
$$

where $P_{l}$ and $Q_{l}$ denote random active and reactive power of load, respectively; $\mu_{p}$ is the mean of active power, and $\varphi_{l}$ the load power factor.

\subsection{Two-point estimate method}

The current research used Hong's 2m PEM method for uncertainty modeling of the renewable energy sources and loads [43]. In case 2m PEM method is used for stochastic problem, two deterministic points on each side of the mean value are found for every random variable. In the next step, the required problem is solved twice for every random variable by generating two deterministic points where the values of other random variables are assumed to be equal to their mean value. Here, the uncertain variables are solar or wind output and load amount. In the first step of $2 \mathrm{~m}$ PEM method, the initial values are calculated as follows:

$$
E(A)^{(1)}=0 ; \quad E\left(A^{2}\right)^{(1)}=0 .
$$

In the second step, two standard locations and probability are expressed in Eqs. (19), (20), (21), and (22), as shown below:

$$
\begin{aligned}
& \xi_{m, 1}=\frac{\lambda_{m, 3}}{2}+{\sqrt{n+\left(\frac{\lambda_{m, 3}}{2}\right)}}^{2} \quad m=1, \ldots, n, \\
& \xi_{m, 2}=\frac{\lambda_{m, 3}}{2}-{\sqrt{n+\left(\frac{\lambda_{m, 3}}{2}\right)}}^{2} \quad m=1, \ldots, n, \\
& P_{m, 1}=\frac{-\xi_{m, 2}}{2 n \sqrt{n+\left(\frac{\lambda_{m, 3}}{2}\right)^{2}}} \quad m=1, \ldots, n, \\
& P_{m, 2}=\frac{-\xi_{m, 1}}{2 n \sqrt{n+\left(\frac{\lambda_{m, 3}}{2}\right)^{2}}} \quad m=1, \ldots, n .
\end{aligned}
$$

Based on these equations, two estimated locations for the $m$ th uncertain variable can be written as:

$$
\begin{aligned}
& x_{m, 1}=\mu_{x, m}+\xi_{m, 1} \cdot \sigma_{x, m}, \\
& x_{m, 2}=\mu_{x, m}+\xi_{m, 2} . \sigma_{x, m},
\end{aligned}
$$

where $\mu_{x, m}$ and $\sigma_{x, m}$ represent the mean and variance of the random variable $m$, respectively.

Thereafter, the objective function is evaluated for the same input parameters and same values for the $m$ th uncertain variable.

The output variable $Y$ can be calculated with respect to input variable $X$ as:

$$
\begin{aligned}
& Y=f(X), \\
& X=\left[\mu_{m, 1}, \mu_{m, 2}, \cdots, x_{m, i}, \cdots, \mu_{m, n}\right] \forall_{i} \in\{1,2\} .
\end{aligned}
$$

For all random variables calculated, the following parameters are given:

$$
\begin{aligned}
& E(Y)^{m+1} \cong E(Y)^{m}+\sum_{i=1}^{2} P_{m, i} . h(X), \\
& E\left(Y^{2}\right)^{m+1} \cong E\left(Y^{2}\right)^{m}+\sum_{i=1}^{2} P_{m, i} \cdot h^{2}(X) .
\end{aligned}
$$

The mean or expected value and standard deviation 
of $Y$ can be expressed as:

$$
\begin{aligned}
\psi_{Y} & =E(Y), \\
\sigma_{Y} & =\sqrt{E\left(Y^{2}\right)-\psi_{Y}^{2}} .
\end{aligned}
$$

\section{SOS algorithm}

SOS is a meta-heuristic algorithm developed by Cheng and Prayogo in 2014. In this algorithm, the symbiotic strategies of the organisms among themselves are used to simulate and survive in the ecosystem. There are three types that have a symbiotic relationship in nature namely the mutualism, commensalism, and parasitism. Based on these relationships, the SOS algorithm can be formulated as follows:

\subsection{Mutualism phase}

Both organisms benefit from each other at the Mutualism phase of SOS. The example of Mutualism is the relationship between bees and flowers. The bees collect nectar and benefit from flowers to make honey. At the time of collecting nectar, bees also take pollen grains from the flower and give them to another flower, which helps pollination. This phase can be expressed by the following equations [44]:

$$
\begin{aligned}
& X_{\text {knew }}=X_{k}+\operatorname{rand}(0,1) \\
& *\left(X_{\text {best }}-\text { Mutual_Vector } * B F_{1}\right), \\
& X_{\text {lnew }}= X_{l}+\text { rand }(0,1) \\
& *\left(X_{\text {best }}-\text { Mutual_Vector } * B F_{2}\right), \\
& \text { Mutual_Vector }=\frac{X_{k}+X_{l}}{2},
\end{aligned}
$$

where $X_{k}$ is the $k$ th member organism of the ecosystem and $X_{l}$ is a random parameter to interact with $X_{k}$. In Eqs. (31) and (32), vector ' $r a n d(0,1)$ ' includes random numbers. $B F_{1}$ and $B F_{2}$ are benefit vectors that are either 1 or 2 . The mutual relation between organisms $X_{k}$ and $X_{l}$ is represented by Mutual_Vector. .

\subsection{Commensalism phase}

In nature, the relationship between two spices in which one collects food from the other without harming or providing benefit to the other is called Commensalism. Such a relationship is found between the remora fish and shark in which remora fish is fed by the leftover of the shark's food by always remaining attached to it which, in turn, brings no benefit or harm to the shark. At this phase, $X_{l}$ is chosen randomly which will interact with $X_{k}$ to create a new organism, $f_{i}^{\max }$, using the following equation:

$$
X_{\text {knew }}=X_{k}+\operatorname{rand}(-1,1) *\left(X_{\text {best }}-X_{l}\right),
$$

where $\left(X_{\text {best }}-X_{l}\right)$ represents the advantage provided to $X_{k}$ by $X_{l}$ to raise its benefit in the ecosystem to the highest degree up to $X_{\text {best }}$.

\subsection{Parasitism phase}

Parasitism is the relationship between two organisms in which once one gets harmed, the other will stand to benefit in the ecosystem. The benefitted organism is called parasite, while the harmed one is known as the host. An example of Parasitism is deer tick attached to the host to suck its blood. However, as a carrier of Lyme disease, it causes joint damage and kidney problems with lack of blood to the host, i.e., deer.

Here, the host in SOS is $X_{l}$ which is selected randomly. An artificial organism called Parasite Vector is created in the search space. When the value of Parasite Vector is larger than $X_{l}$, the position of $X_{l}$ will be taken by the parasite. On the contrary, in case of the higher fitness of $X_{l}$, the Parasite Vector will not survive in the ecosystem.

\section{SOS-based optimal placement and sizing DG}

For determining the optimal location and sizing of DG based on SOS in the distribution system, the following steps should be taken:

Step 1. Initialize ecosystem with eco size, number of iterations, termination criteria, and maximum number of fitness function evaluations (max-fit-eval);

Step 2. Initialize a number of DGs and power output of each DG by assigning the maximum and minimum output of each DG;

Step 3. Create an initial ecosystem by determining the random location and size of DG;

Step 4. Run the load flow and check the constraints. If the constraints are satisfied, go to the next step; otherwise, create the ecosystem again;

Step 5. Continue the outer loop up to the maximum value of iteration while the inner loop will do work until the last member of ecosystem is reached;

Step 6. At the Mutualism phase, choose an organism $X_{l}$ such that $X_{l} \neq X_{k}$;

Step 7. Determine the Mutual-Vector and Benefit Factor (BF);

Step 8. Modify $X_{k}$ and $X_{l}$ based on Eqs. (31) and (32);

Step 9. Calculate the fitness value of the modified organism. If the modified organism has a higher fitness value, it will replace the old one;

Step 10. At the commensalism phase, select an organism $X_{l}$ randomly such that $X_{l} \neq X_{k}$; 
Step 11. Modify $X_{k}$ using $X_{l}$ based on Eq. (34);

Step 12. Calculate the fitness value of the new organism. If the fitness value of the new organism is higher than that of others, it will be retained in the ecosystem;

Step 13. At the parasitism phase, select an organism $X_{l}$ randomly such that $X_{l} \neq X_{k}$;

Step 14. Parasite-Vector is created from organism $X_{l}$;

Step 15. Calculate the fitness value of the Parasite Vector and if the fitness value is higher than $X_{l}$, the position of $X_{l}$ will be replaced by Parasite Vector;

Step 16. Repeat the steps for eco size;

Step 17. To achieve the termination criteria, stop the process and find an optimal solution to the singleobjective optimization problem; otherwise, repeat the steps from Steps 6 to 16 . In the case of the multiobjective problem, when the current iteration exceeds or is equal to the maximum number of iterations, the result will be stored in an array (Pareto-optimal set) and the iteration will stop; otherwise, repeat the steps from Steps 6 to 16 ;

Step 18. For bi-objective problem, the value of will vary from 0 to 1 in the steps of 0.05 ; in Steps 3 to 17 , it will be repeated until the value of reaches 1 ;

Step 19. In the proposed technique for the biobjective optimization problem, different Paretooptimal solutions are obtained using different weights and then, the best compromise result is selected from the optimal Pareto set. The two objective functions have different ranges and dimensions. As a result, a fuzzy satisfying method is utilized to normalize the objective functions.

In order to solve the multi-objective optimization problem, the best result from the Pareto-optimal front was selected using the fuzzy membership technique, which maps the value of the objective function into the interval $[0,1]$. For the $i$ th objective function, the fuzzy membership function $K_{f i}$ can be written as follows:

$$
K_{f i}= \begin{cases}0, K_{i} & \leq 0 \\ K_{i}, & 0<K_{i}<1 \\ 1, & K_{i} \geq 1\end{cases}
$$

where:

$$
K_{i}=\frac{f_{i}^{\max }-f_{i}}{f_{i}^{\max }-f_{i}^{\min }},
$$

where $f_{i}^{\min }$ and $f_{i}^{\max }$ are the minimum and maximum values of the $i$ th objective function, respectively. The normalized membership function for each solution $j$ can be written as:

$$
F D M^{j}=\left[\frac{\sum_{i=1}^{n} K_{f i}^{j}}{\sum_{j=1}^{M} \sum_{i=1}^{n} K_{f i}^{j}}\right],
$$

where $M$ and $n$ are the values of the non-dominated solutions and number of objective functions, respectively. The best compromise solution is found for the maximum value of $F D M^{j}$. Figure 1 illustrates the SOS-based optimal DG placement technique.

\section{Simulation results and discussion}

SOS algorithm used for determining the best location and DG rating was simulated in MATLAB and tested on two different distribution networks, i.e., IEEE 33 bus radial distribution system (Test System-I) and IEEE 69 bus radial distribution system (Test SystemII). In both networks, three DGs are installed using the optimization algorithm, and the DGs are considered Type-1 DG; in other words, they can inject real power only. The maximum sizes of all DGs are considered as 1.5 MW. The effect of DG installation on the voltage profile, VSI, and voltage security state of the distribution system was also analyzed in the present study under all single line contingencies. In this analysis, those buses that do not get power due to particular contingency were eliminated from the system; for example, tripping the line between buses 3 and 23 as well as between buses 24 and 25 does not get power for Test System-I. Therefore, buses 24 and 25 are excluded from the reconfigured network for analysis. A similar technique is applicable to every line tripping in all test systems.

This study took into account three different cases for optimal location and sizing of three DGs. Case 1 and Case 2 focus on the voltage profile and VSI improvements, respectively, and Case 3 is a multiobjective problem that simultaneously takes into account the voltage profile and VSI improvements. Initially, all the three cases are modeled without considering the solar, wind, or load uncertainties. In the next part, the effect of uncertain parameters on the objective functions of the distribution systems is discussed. Upon using the SOS-based renewable DG integration in the test systems, Weibull PDF and Beta PDF are generated for every time section, considering that uncertainty requires historical hourly wind speed data and historical hourly solar irradiance data, respectively. It is also assumed that the buses within the test system are geographically so close to each other that the solar data, wind data, and their corresponding distributions are also the same for all buses. In this study, the cut-in, rated, and cut-out speeds of the wind DG are 


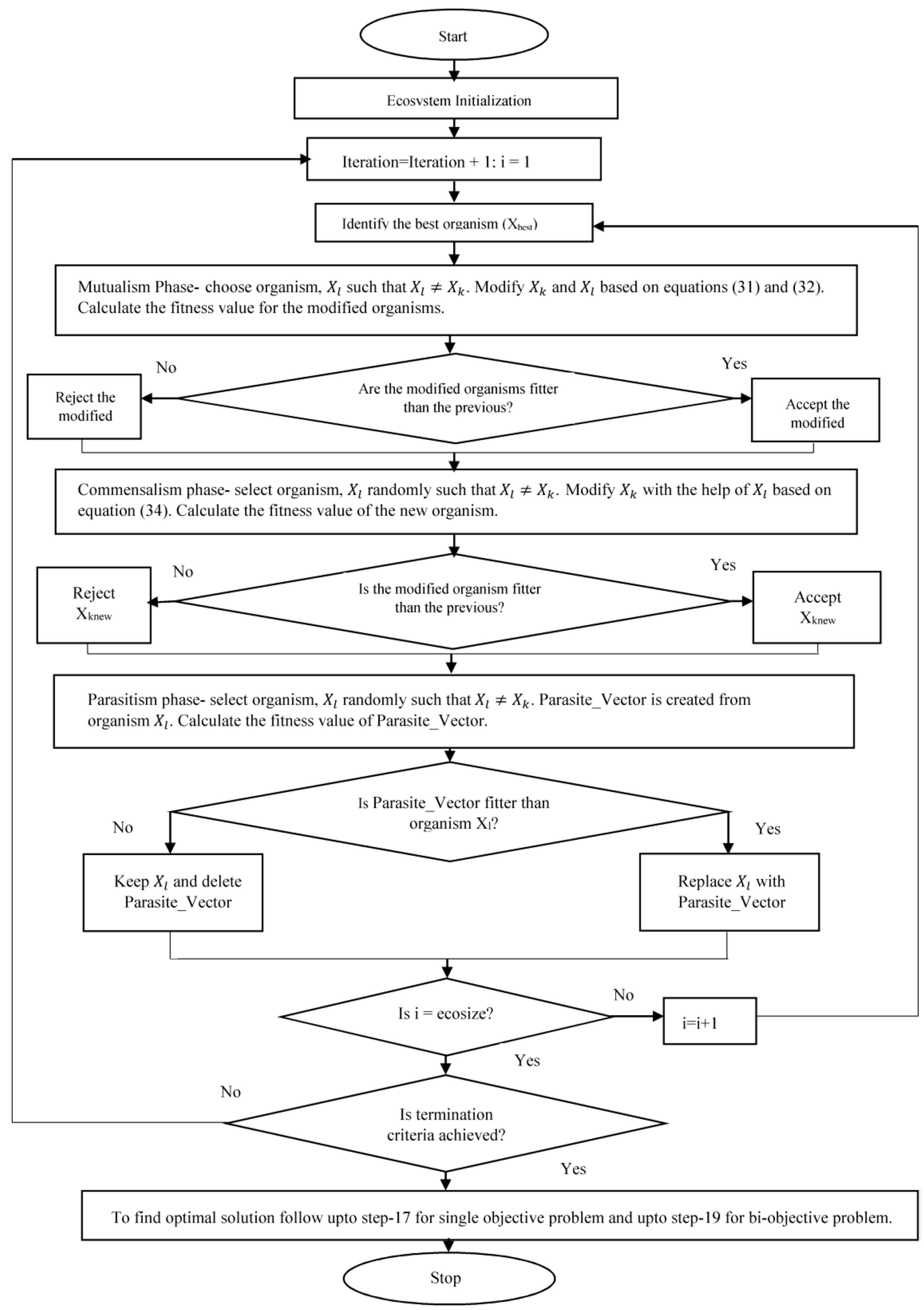

Figure 1. Algorithm of the DG placement by SOS. 
considered as $3 \mathrm{~m} / \mathrm{s}, 11.5 \mathrm{~m} / \mathrm{s}$, and $20 \mathrm{~m} / \mathrm{s}$, respectively. The scale parameter $c$ and shape parameter $K$ used for Weibull distribution are measured as 8.78 and 1.75 , respectively. For Beta distribution, the values of the shape parameters $\alpha$ and $\beta$ were obtained as 6.38 and 3.43 , respectively. The rated output power of all the three DGs is $1.5 \mathrm{MW}$, and they operate at unity power factor. Load demand was modeled in this paper using normal distribution, considering $5 \%$ standard deviation from mean value.

\subsection{Test System-I: IEEE 33 bus radial distribution system}

Test System-I has reactive power demand of $2300 \mathrm{kVAr}$, active power demand of $3715 \mathrm{~kW}, 33$ buses, and 32 lines. The base $\mathrm{kV}$ and base MVA values are $11 \mathrm{kV}$ and 100 MVA, respectively. The data table was extracted from the referenced study [45]. Figure 2 shows the oneline diagram of IEEE 33 bus radial distribution system. In [37], the performance of Test System-I was evaluated without installing DG in the system with the voltage deviation of 0.1338 p.u and $\mathrm{VSI}^{-1}$ of 1.4988 .

\subsubsection{Objective functions evaluation without considering uncertainty}

For performance analysis of Test System-I, three different cases were studied based on the three objective functions. In Case 1, the locations and sizes of DGs were determined based on the maximization of bus voltage profile as the objective function. In Case 2, VSI improvement was considered the objective function. A multi-objective function was evaluated in Case 3

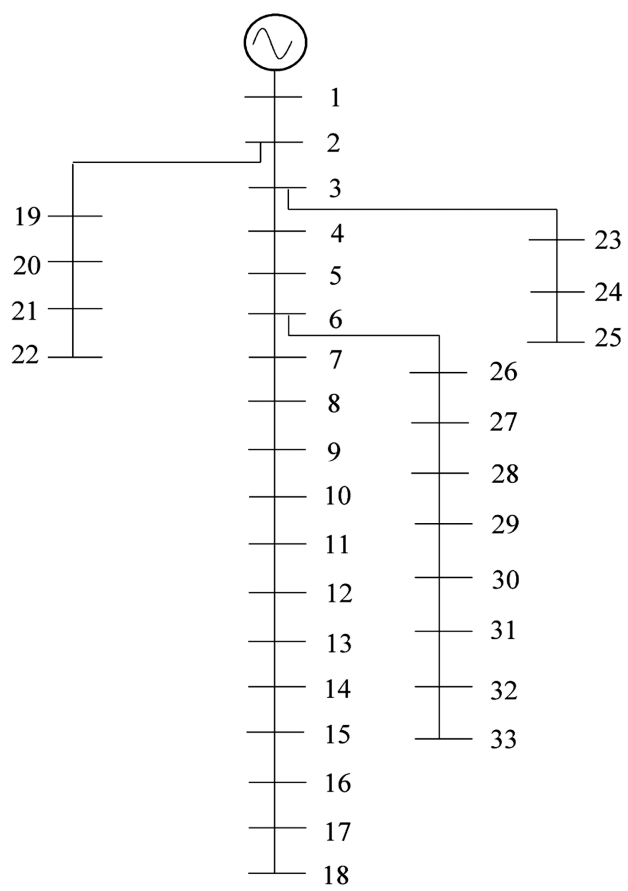

Figure 2. One-line diagram of IEEE 33 bus radial distribution system. which aimed to find a compromise solution between maximization of bus voltage profile and improvement of VSI.

Case 1: Improvement of voltage profile. According to the SOS algorithm in Case 1, buses 7, 13 , and 31 are the most excellent locations for DG placement with rating values of $1.5 \mathrm{MW}, 0.9535 \mathrm{MW}$, and 1.2631 MW, respectively, as shown in Table 1. The bus numbers and DG sizes by QOSIMBO-Q and SIMBO-Q are also shown in Table 1.

In this case, the objective functions for voltage profile improvement obtained from the SOS, QOSIMBO-Q, and SIMBO-Q algorithms were 0.000656 p.u, 0.00066 p.u, and 0.00075 p.u, respectively, as shown in Figure 3. In addition, the optimal DG placement using the SOS algorithm delivers the best results, compared to QOSIMBO-Q and SIMBO$\mathrm{Q}$, since the objective function value offered by SOS is the smallest among the others. Although voltage profile improvement is considered as the objective function in Case 1, optimal allotment of DG also affects the other objective function namely $\mathrm{VSI}^{-1}$ and its values for SOS, QOSIMBO-Q, and SIMBO$\mathrm{Q}$ algorithms were obtained as 1.0685, 1.0685, and 1.0711 , respectively. In this case study, the voltage deviation decreased from 0.1338 p.u to 0.000656 p.u while the $\mathrm{VSI}^{-1}$ value decreased from 1.4988 to 1.0685 through the SOS method.

Figure 4 represents the convergence graph of the voltage deviation obtained from SOS algorithm in the case of DG placement in Test System-I. This graph shows that the SOS algorithm converges to very small numbers of iterations.

Table 1. Simulation results of the voltage profile improvement for Test System-I.

\begin{tabular}{lcc}
\hline \multicolumn{1}{c}{ Method } & Bus no. & DG size (MW) \\
\hline SOS & $7,13,31$ & $1.5000,0.9535,1.2631$ \\
QOSIMBO-Q [26] & $7,13,31$ & $1.4903,0.9580,1.2714$ \\
SIMBO-Q [26] & $7,31,13$ & $1.0608,1.4418,1.0558$ \\
\hline
\end{tabular}

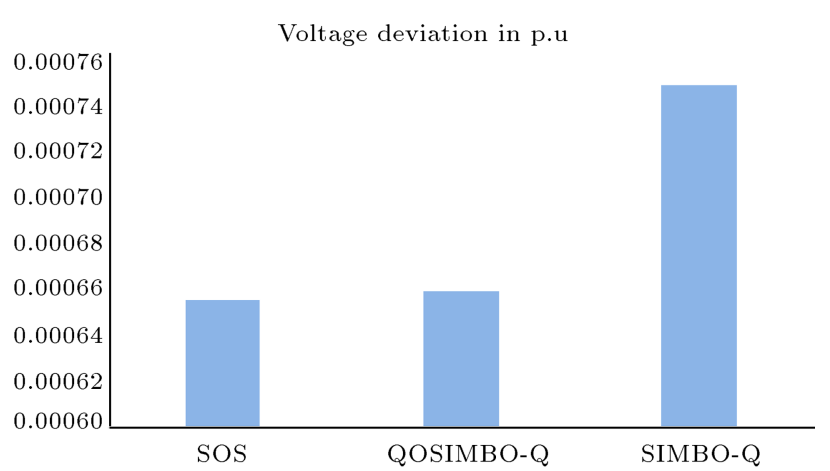

Figure 3. Comparison of the voltage deviation by SOS, QOSIMBO-Q, and SIMBO-Q in Test System-I. 


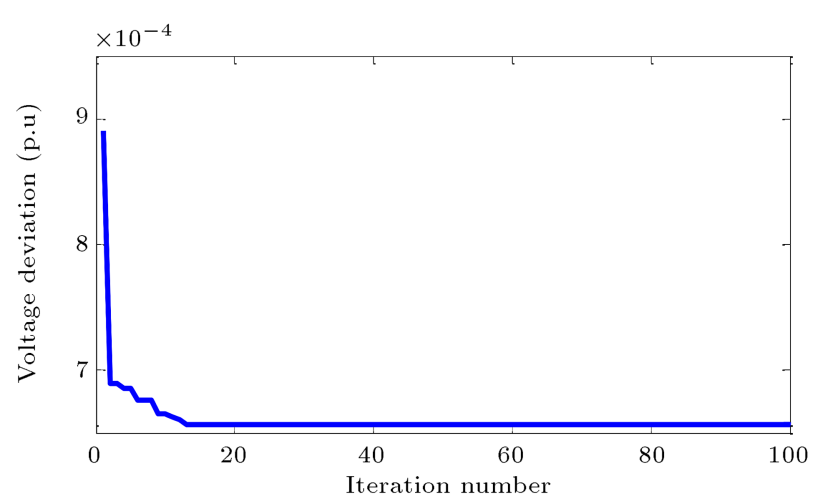

Figure 4. Convergence curve of the voltage deviation by SOS algorithm in Test System-I.

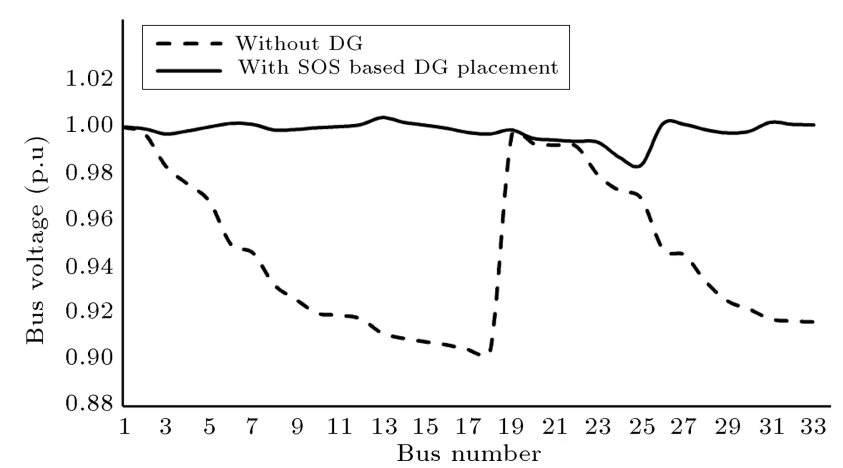

Figure 5. Voltage profile change of the Test System-I with installation of DG by SOS.

Figure 5 shows the effects of installing DG using SOS algorithm on the bus voltage profile of Test System-I. Clearly, with proper allotment of DG, the minimum voltage magnitude for the system can be improved from 0.8820 p.u (at bus 18 ) to 0.9836 p.u (at bus 25) under base configurations. Voltage magnitudes of all buses are also improved due to the DG placement via SOS technique.

Case 2: Improvement of VSI. In case the SOS algorithm is used for VSI improvement, buses 12, 31, and 25 are the appropriate locations for DG placement with rating of $1.5 \mathrm{MW}, 1.5 \mathrm{MW}$, and 0.7095 MW, respectively. Through the QOSIMBO-Q and SIMBO-Q algorithms, the optimal location and size of DGs can also be calculated, as shown in Table 2 .

The values for the objective functions in this case in the SOS, QOSIMBO-Q, and SIMBO-Q algo-

Table 2. Simulation results of the VSI improvement for Test System-I.

\begin{tabular}{lcc}
\hline \multicolumn{1}{c}{ Method } & Bus no. & DG size (MW) \\
\hline SOS & $12,31,25$ & $1.5000,1.5000,0.7095$ \\
QOSIMBO-Q [26] & $12,25,31$ & $1.5000,0.7199,1.5000$ \\
SIMBO-Q [26] & $16,25,33$ & $1.4866,0.6873,1.4995$ \\
\hline
\end{tabular}

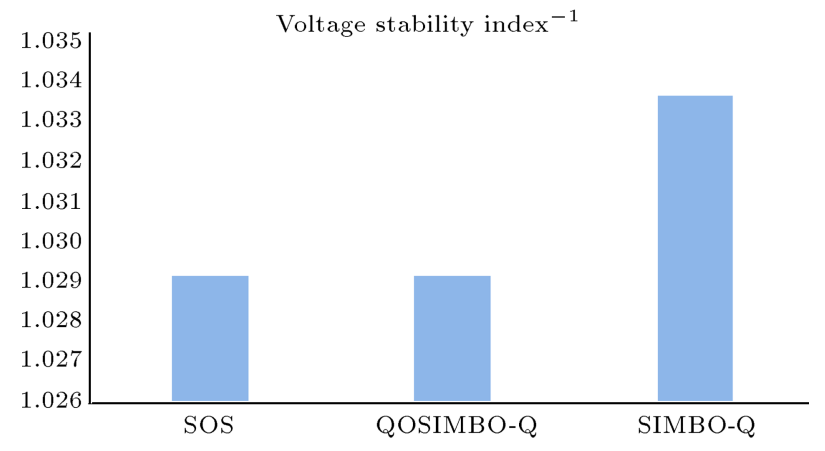

Figure 6. Comparison of inverse of VSI values by SOS, QOSIMBO-Q, and SIMBO-Q in Test System-I.

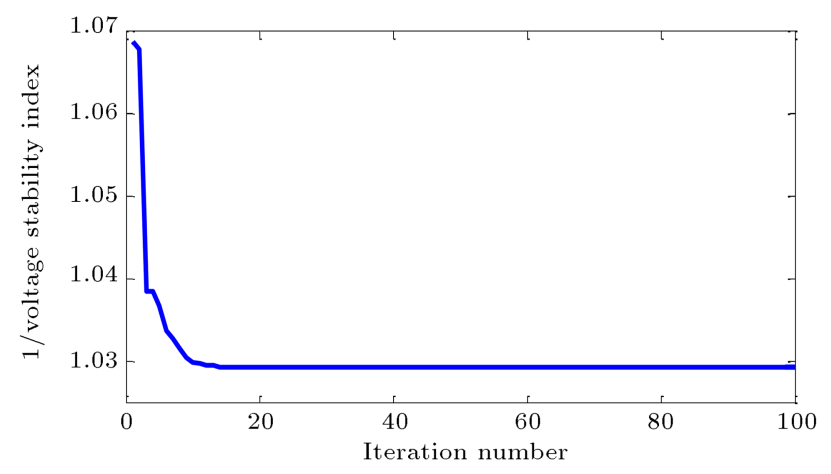

Figure 7. Convergence characteristics of $\mathrm{VSI}^{-1}$ of Test System-I by SOS.

rithms were $1.02918,1.02920$, and 1.03370 , respectively, as depicted in Figure 6. As observed in this figure, SOS algorithm has the lowest objective function value, compared to the QOSIMBO-Q and SIMBO-Q algorithms. In Case 2, the voltage deviation values resulting from the $\mathrm{DG}$ installation through the SOS, QOSIMBO-Q, and SIMBO-Q algorithms were 0.00066 p.u, 0.00066 p.u, and 0.00310 p.u, respectively. SOS-based DG placement decreased voltage deviation and $\mathrm{VSI}^{-1}$ values from $0.1338 \mathrm{p} . \mathrm{u}$ to 0.00066 p.u and from 1.4988 to 1.02918 , respectively.

Figure 7 illustrates the convergence graph of the $\mathrm{VSI}^{-1}$ obtained from SOS algorithm in the DG placement in Test System-I according to which it can be deduced that the SOS algorithm converges to very small numbers of iterations.

Case 3: Improvement of voltage profile and VSI. Based on the simulation result of the bi-objective case, which ensured simultaneous voltage profile and VSI improvements, buses 31, 25, and 12 were selected as the optimum locations of DG placement with the DG sizes of $1.5 \mathrm{MW}, 0.7089$ MW, and 1.5 MW, respectively, as shown in Table 3. This bi-objective problem can be solved by the SOS algorithm for Test System-I, which in turn satisfied all the constraints mentioned in Subsection 2.2. Table 3 makes a comparison between the results 
Table 3. Simulation results of the bio-objective case for Test System-I.

\begin{tabular}{lcc}
\hline \multicolumn{1}{c}{ Method } & Bus no. & DG size (MW) \\
\hline SOS & $31,25,12$ & $1.5000,0.7089,1.5000$ \\
QOSIMBO-Q [26] & $12,25,31$ & $1.5000,0.7199,1.5000$ \\
SIMBO-Q [26] & $25,33,12$ & $0.7160,1.5000,1.5000$ \\
\hline
\end{tabular}

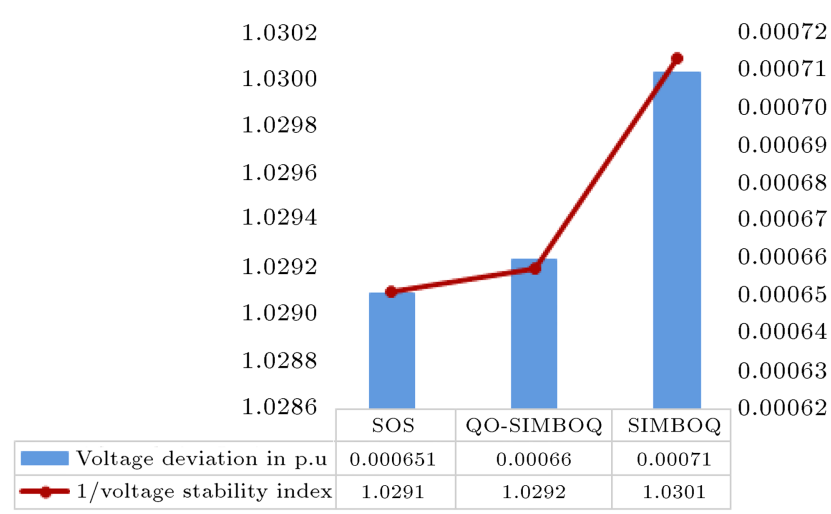

Figure 8. Comparison of voltage deviation and inverse of VSI by SOS, QOSIMBO-Q, and SIMBO-Q in Test System-I.

obtained from SOS and those from QOSIMBO-Q and SIMBO-Q.

The best compromise solution values obtained from the SOS algorithm for voltage profile and VSI improvements were 0.000651 p.u and 1.0291, respectively. These values were then compared with the results from QOSIMBO-Q and SIMBO-Q in Figure 8 according to which it can be stated that the compromise solution obtained from the SOS algorithm was better than those obtained from the QOSIMBO-Q and SIMBO-Q algorithms.

Figure 9 presents the pareto-optimal front obtained from the SOS algorithm to facilitate the simultaneous voltage profile and VSI improvements in the three DGs functioning based on the unity power factor.

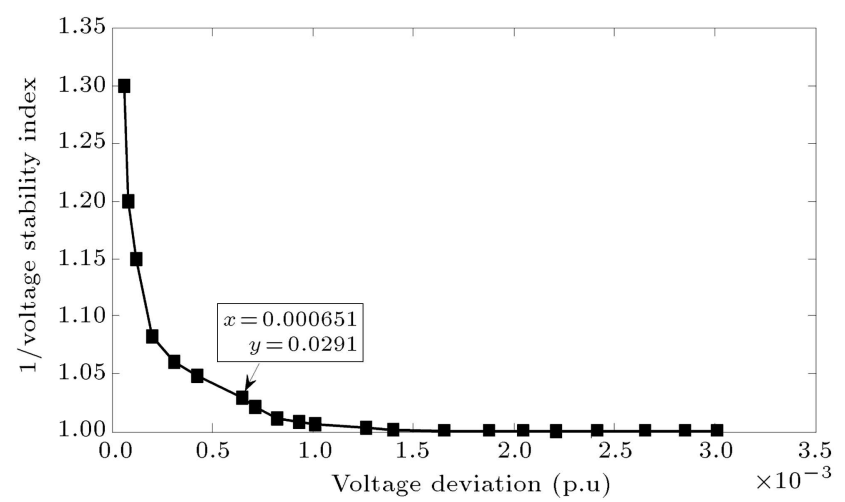

Figure 9. Pareto-optimal front by SOS for voltage profile and VSI improvement in Test System-I.
According to this graph, the best compromise solution was obtained from the SOS algorithm when the values of voltage deviation and VSI $^{-1}$ were 0.000651 p.u and 1.0291, respectively.

\subsubsection{Objective functions evaluation considering uncertainty}

Given the stochastic nature of wind and solar power and variations in the load demands, probabilistic analysis is highly recommended. In this section, 2m PEM method in coordination with SOS technique remodels all the cases mentioned in the previous subsections. In this test system, three DGs are to be installed, which are renewable (wind or solar). Therefore, their outputs are uncertain and for this reason, the output powers of the three DGs are three uncertain variables. At the same time, the test system has 32 load buses with uncertain loads; hence, there are 32 uncertain load parameters. Here, there are entirely $35(32+3)$ uncertain parameters and $70(2 \times 35)$ deterministic points for finding the optimal solution since $2 \mathrm{~m}$ PEM method calculates two deterministic points on each side of the mean value for every random variable.

Case 1: Improvement of voltage profile. Upon using 2m PEM method and SOS in the Test System$\mathrm{I}$, the mean and standard values of voltage deviation in Case 1 were measured as 0.0051 and 0.0050 , respectively. In this case, the optimal buses for DG allocation are buses 33, 12, and 9 .

Case 2: Improvement of VSI. Based on a combination of $2 \mathrm{~m}$ PEM method and SOS, the mean and standard deviation values of $\mathrm{VSI}^{-1}$ in Case 2 were 1.1124 and 0.0441 , respectively, where the candidate buses for DG allotment are buses 11, 6, and 33 .

Case 3: Improvement of voltage Profile and VSI. In Case 3, a compromise solution was found between the voltage profile and VSI improvements by modeling the uncertainties in the SOS program. The mean and standard deviation values for the voltage profile improvement were obtained as 0.0051 and 0.0050 , respectively, and the mean and standard deviation values for VSI improvement were 1.0942 and 0.0349 , respectively. In this case, the candidate buses for DG allocation are buses 9, 33, and 12 . Table 4 presents the simulation results for Cases 1 and 3 .

\subsubsection{Effect of DG placement on system's voltage security state}

Given that the optimal allocation of DGs in the distribution system can improve the VSI of the distribution system, it further improves the voltage security state of the very system. At every single line contingency, the VSI values of the system are calculated for Test 
Table 4. SOS-based simulation results of the Test System-I considering uncertainty.

\begin{tabular}{cccccc}
\hline Case & $\begin{array}{c}\text { Mean of voltage } \\
\text { deviation }\end{array}$ & $\begin{array}{c}\text { Standard deviation } \\
\text { of voltage deviation }\end{array}$ & $\begin{array}{c}\text { Mean of } \\
\text { VSI }^{-1}\end{array}$ & $\begin{array}{c}\text { Standard deviation } \\
\text { of VSI }^{-1}\end{array}$ & $\begin{array}{c}\text { Candidate buses for } \\
\text { DG allocation }^{\text {1 }}\end{array}$ \\
\hline 1 & 0.0051 & 0.0050 & - & - & $33,12,9$ \\
2 & - & - & 1.1124 & 0.0441 & $11,6,33$ \\
3 & 0.0051 & 0.0050 & 1.0942 & 0.0349 & $9,33,12$ \\
\hline
\end{tabular}

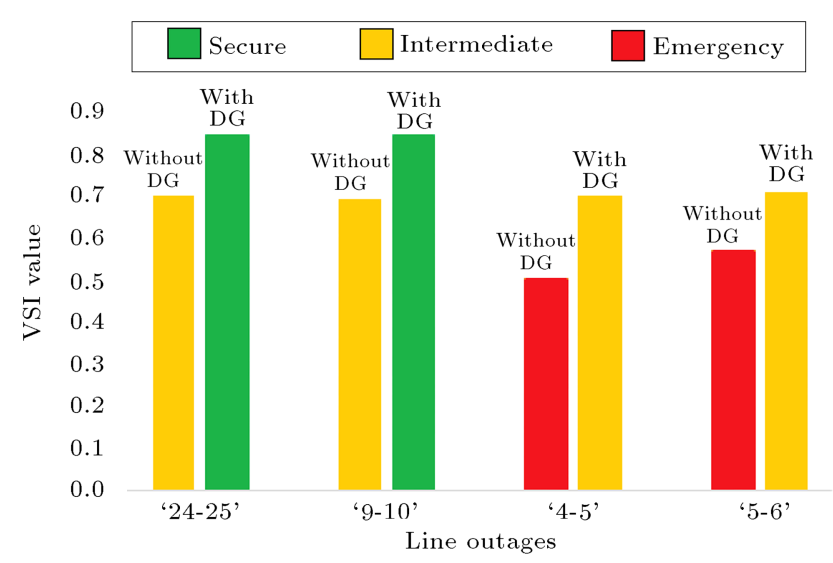

Figure 10. Change of the voltage security state due to the optimal placement of DG by SOS in Test System-I.

System-I. Natural Breaks methodology [46] is employed to classify the VSI values into three categories by choosing the suitable group split as there is the least divergence in side every data set and highest dissimilarity among groups. These three categories will identify three classes of the operating states of the distribution system, namely Secure $(>0.7185$ and $<1.00)$, Intermediate (0.6950-0.7185), and Emergency $(<0.6950)$ states. For some line contingencies after DG allocation, the values of the VSI of the system may be improved such that the operating states of the system will go to the next category. For some contingencies, the system VSI value and operating states (before and after DG allocation) are depicted in Figure 10, showing that proper DG allocation via SOS technique improves the VSI value as well as the operating states of the power network. For this reason, the operating states in the intermediate state earlier are now shifted to secure states and similarly, in the emergency state, they are now taken to the secure state.

\subsection{Test System-II: IEEE 69 bus radial distribution system}

Test System-II has reactive power load of $2694.1 \mathrm{kVAr}$, active power load of $3791.89 \mathrm{~kW}, 69$ buses, and 68 lines. The base $\mathrm{kV}$ and base MVA are $11 \mathrm{kV}$ and 100 MVA, respectively. The data of the system are available in Reference [47]. The one-line diagram of IEEE 69 bus radial distribution system is shown in Figure 11. The objective function value of voltage deviation is 0.0993 and that of $\mathrm{VSI}^{-1}$ is 1.4635 [37].

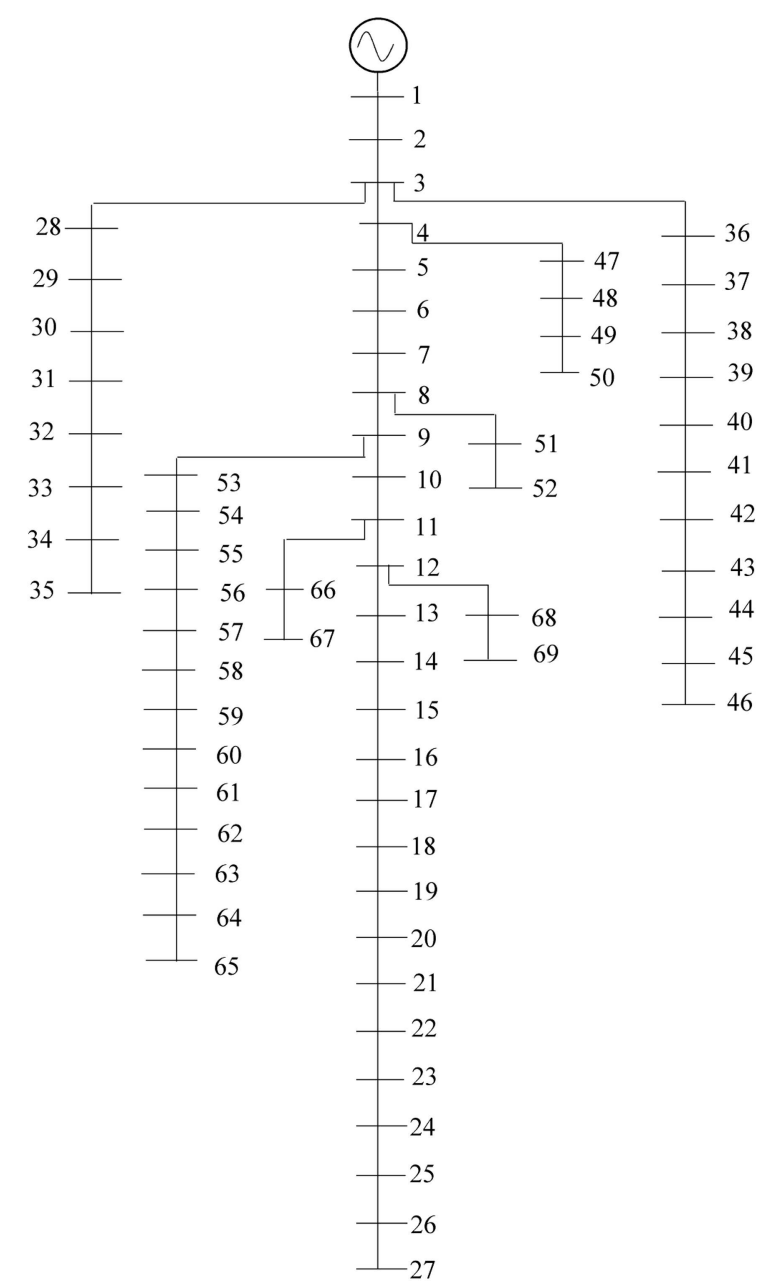

Figure 11. One-line diagram of IEEE 69 bus radial distribution system.

\subsubsection{Objective functions evaluation without considering uncertainty}

The performance of Test System-II is evaluated under three different cases. Case 1 is modeled with SOS for improvement of voltage profile; Case 2 is simulated for the improvement of VSI, whereas Case 3 is a multi-objective problem that considers voltage profile improvement and VSI improvement simultaneously.

Case 1: Improvement of voltage profile. For three DGs installation in case of voltage profile improvement objective function, the optimal size and location are obtained using SOS algorithm. Buses 14,63 , and 57 are found as the best possible nodes 
Table 5. Simulation results of the voltage profile improvement for Test System-II.

\begin{tabular}{lcc}
\hline \multicolumn{1}{c}{ Method } & Bus no. & DG size (MW) \\
\hline SOS & $14,63,57$ & $0.8827,1.5000,1.1293$ \\
QOSIMBO-Q [26] & $14,57,63$ & $0.8777,1.1559,1.4914$ \\
SIMBO-Q [26] & $63,14,57$ & $1.5000,0.9011,1.1320$ \\
\hline
\end{tabular}

of the network to place DGs and the ratings are $0.8827 \mathrm{MW}, 1.5000 \mathrm{MW}$, and $1.1293 \mathrm{MW}$, as shown in Table 5. The locations and sizes of DGs obtained by QOSIMBO-Q and SIMBO-Q are also shown in Table 5 .

A comparison between different techniques for voltage deviation minimization is made, as shown in Figure 12, revealing that SOS-based technique obtained the greatest outcome among the other algorithms such as QOSIMBO-Q and SIMBO-Q in terms of delivering the lowest value of voltage deviation. Here, the value of $\mathrm{VSI}^{-1}$ obtained by SOS, QOSIMBO-Q, and SIMBO-Q is 1.0235.

The convergence graph of voltage deviation for Test System-II is shown in Figure 13 from which it is seen that SOS algorithm converges very fast to give the optimal solution.

The voltage magnitudes of all the buses of Test System-II before DG installation and after DG installation by SOS technique are compared in Fig-

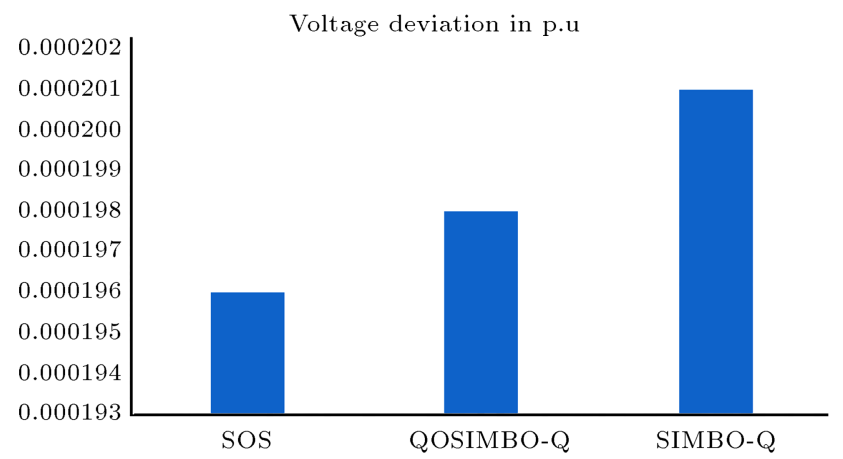

Figure 12. Comparison of the voltage deviations by SOS, QOSIMBO-Q, and SIMBO-Q in Test System-II.

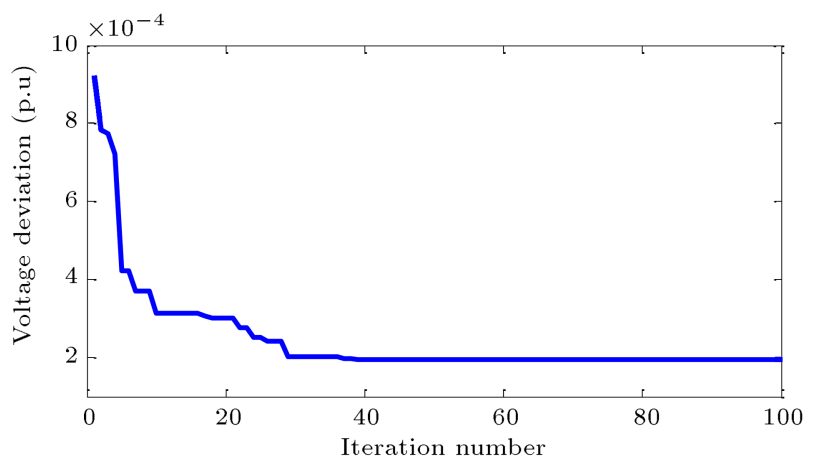

Figure 13. Convergence curve of the voltage deviation based on SOS algorithm in Test System-II.

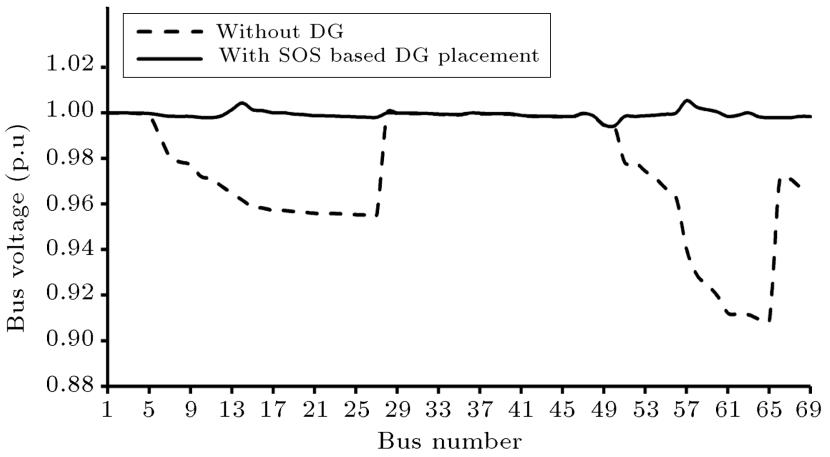

Figure 14. Voltage profile change of the Test System-II followed by installation of DG by SOS.

Table 6. Simulation results of the VSI improvement for Test System-II.

\begin{tabular}{lcc}
\hline \multicolumn{1}{c}{ Method } & Bus no. & DG size (MW) \\
\hline SOS & $53,14,61$ & $1.3271,0.8929,1.5000$ \\
QOSIMBO-Q [26] & $58,12,61$ & $0.8465,1.5000,1.4534$ \\
SIMBO-Q [26] & $12,58,61$ & $1.5000,0.8000,1.5000$ \\
\hline
\end{tabular}

ure 14, showing that there is a definite increase in bus voltage magnitude after DG placement by SOS. Due to SOS-based DG installation, the minimum value of bus voltage magnitude of the network, which was earlier 0.8776 p.u (at bus number 65 under base configuration), improved to 0.9942 p.u (at bus 50).

Case 2: Improvement of VSI. In this case, SOS algorithm is used to find the optimal locations and size of 3 DGs where the main aim is to improve VSI. From the result of Case 2, the optimal locations of DG placement include buses 53, 14, and 61 with ratings of $1.3271 \mathrm{MW}, 0.8929 \mathrm{MW}$, and $1.5000 \mathrm{MW}$, respectively. A comparison among SOS, QOSIMBO$\mathrm{Q}$, and SIMBO-Q regarding DG size and location is presented in Table 6 .

The value of $\mathrm{VSI}^{-1}$ obtained by SOS is 1.00210 , whereas the value obtained by QOSIMBO-Q and SIMBO-Q is 1.02350, as shown in Figure 15. Among SOS, QOSIMBO-Q, and SIMBO-Q, it is found that

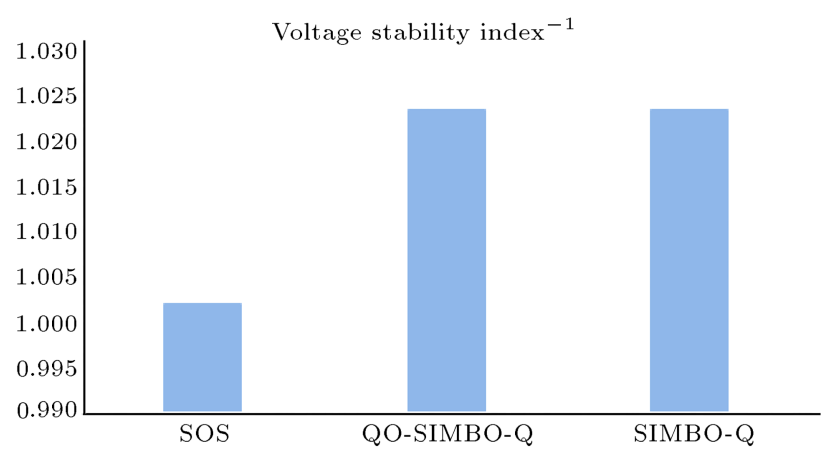

Figure 15. Comparison of inverse of VSI by SOS, QOSIMBO-Q and SIMBO-Q in Test System-II. 


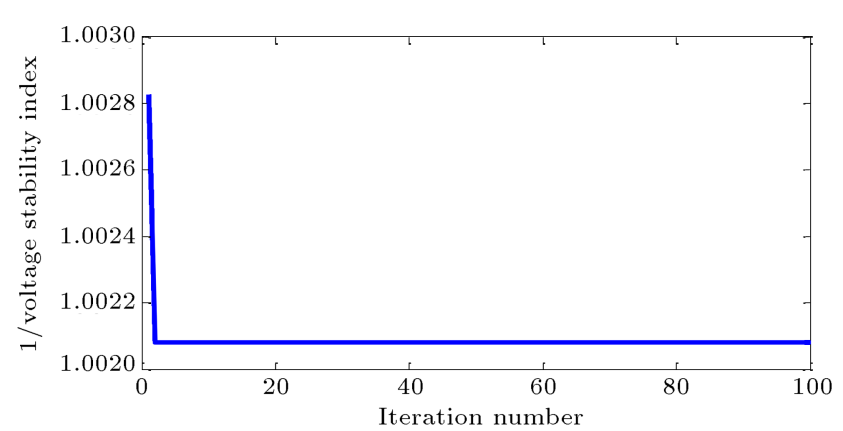

Figure 16. Convergence characteristics of $\mathrm{VSI}^{-1}$ of Test System-II by SOS.

the proposed SOS technique gives the best result out of the other established algorithms. When DGs are placed based on SOS, QOSIMBO-Q, and SIMBO$\mathrm{Q}$ algorithms for minimization of $\mathrm{VSI}^{-1}$, the value of voltage deviation offered by SOS is 0.00038 p.u, whereas the value as 0.00049 p.u is determined by QOSIMBO-Q and SIMBO-Q.

Figure 16 shows the convergence graph of $\mathrm{VSI}^{-1}$ using the SOS algorithm in Test System-II, which shows high convergence rate of the SOS algorithm in case of DG placement using improvement of VSI as the objective function.

Case 3: Improvement of voltage profile and VSI. Table 7 lists the candidate buses for DG allocation along with the DG sizes determined through the SOS, QOSIMBO-Q, and SIMBO-Q algorithms for the bi-objective problem.

The voltage profile and VSI improvement values of the best compromise solution obtained from the SOS were obtained as 0.00024 p.u and 1.02348 , respectively, which were then compared with the results from QOSIMBO-Q and SIMBO-Q algorithms, as shown in Figure 17. In this figure, the compromise result obtained from the SOS is best compared to those from other techniques.

Figure 18 shows the Pareto-optimal front obtained through the SOS for Case 3 of Test System-II according to which the SOS technique can offer an optimal solution in Case 3 when the values of voltage deviation and $\mathrm{VSI}^{-1}$ are 0.00024 p.u and 1.02348, respectively.

\subsubsection{Objective functions evaluation considering uncertainty}

There are 68 load buses with uncertain loads in the

Table 7. Simulation results of the bi-objective case for Test System-II.

\begin{tabular}{lcc}
\hline \multicolumn{1}{c}{ Method } & Bus no. & DG size (MW) \\
\hline SOS & $58,15,3$ & $0.6745,1.5000,0.5992$ \\
QOSIMBO-Q [26] & $62,57,13$ & $1.4703,1.1272,1.1113$ \\
SIMBO-Q [26] & $13,57,62$ & $1.1231,1.1276,1.5000$ \\
\hline
\end{tabular}

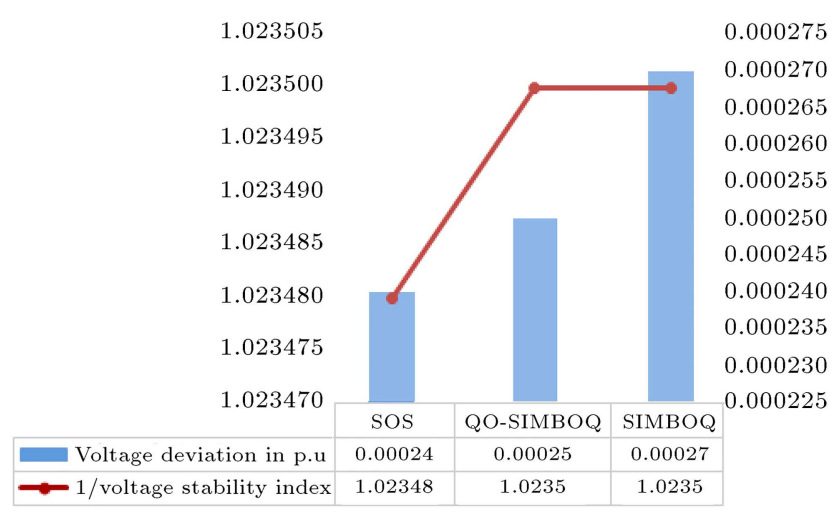

Figure 17. Comparison between voltage deviation and inverse of VSI by SOS, QOSIMBO-Q, and SIMBO-Q in Test System-II.

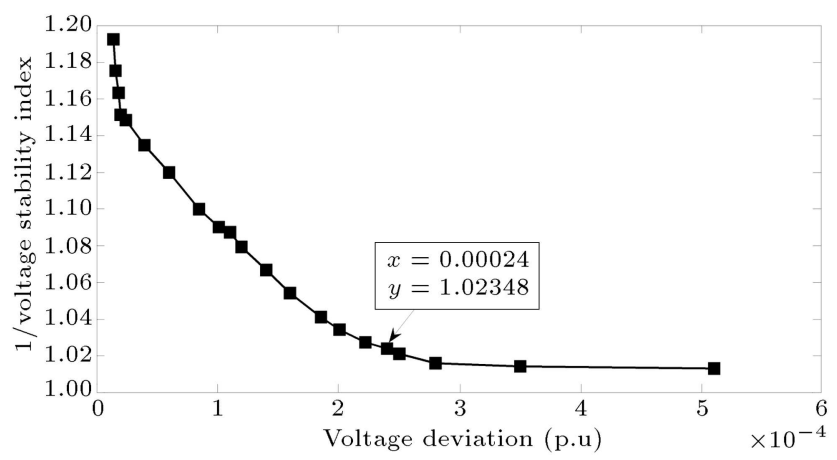

Figure 18. Pareto-optimal front by SOS for voltage profile and VSI improvement in Test System-II.

Test System-II, and three DGs will be installed on the system by optimization of the uncertain output power. Therefore, a total of $71(68+3)$ uncertain variables are available in the Test System-II.

Case 1: Improvement of voltage profile. Upon adding 2m PEM method-based uncertainty modeling with SOS program, the mean and standard deviations of the voltage deviation can be calculated as 0.0017 and 0.0016 , respectively. In this case, the candidate buses for DG allocation are buses 13, 57, and 63 .

Case 2: Improvement of VSI. Considering the uncertainty of solar, wind, and load demand, SOS method finds the appropriate buses for DG allocation, i.e., buses 53, 15, and 61. The mean and standard deviation of the $\mathrm{VSI}^{-1}$ are 1.0123 and 1.0052, respectively.

Case 3: Improvement of voltage profile and VSI. The mean and standard deviation for the voltage deviation in the best compromise solution obtained from the SOS, considering uncertainty in bi-objective problem, were obtained as 0.0016 and 0.0015 , respectively, while the corresponding values for $\mathrm{VSI}^{-1}$ were 1.0045 and 1.0048 , respectively. In this case, buses 63,13 , and 57 were selected as the 
Table 8. SOS-based simulation results of Test System-II considering uncertainty.

\begin{tabular}{cccccc}
\hline Case & $\begin{array}{c}\text { Mean of voltage } \\
\text { deviation }\end{array}$ & $\begin{array}{c}\text { Standard deviation } \\
\text { of voltage deviation }\end{array}$ & $\begin{array}{c}\text { Mean of } \\
\text { VSI }^{-1}\end{array}$ & $\begin{array}{c}\text { Standard deviation } \\
\text { of VSI }\end{array}$ & $\begin{array}{c}\text { Candidate buses for } \\
\text { DG allocation }^{-1}\end{array}$ \\
\hline 1 & 0.0017 & 0.0016 & - & - & $13,57,63$ \\
2 & - & - & 1.0123 & 1.0052 & $53,15,61$ \\
3 & 0.0016 & 0.0015 & 1.0045 & 1.0048 & $63,13,57$ \\
\hline
\end{tabular}

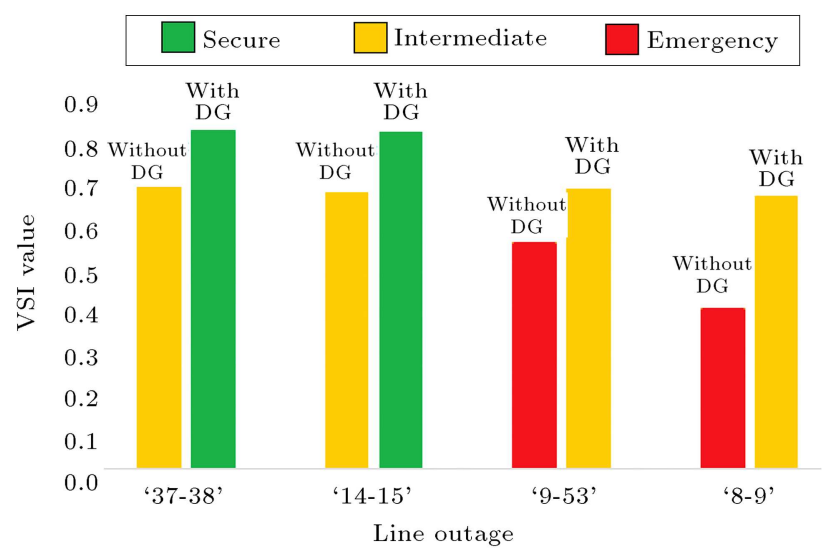

Figure 19. Variation of the voltage security state due to optimal placement of DG by SOS in Test System-II.

appropriate buses for DG allocation. Table 8 shows the results of Cases $1-3$.

\subsubsection{Effect of DG placement on system's voltage security state}

In case of line contingency in the presence of DGs, the system will be in a more secure position than the situation when the system is run without DG. To demonstrate the impact of DG placement on Test System-II, the VSI values of the system are calculated at every single line contingency and depending on these values, the system is classified into three states namely Secure $(>0.7387$ and $<1.00)$, Intermediate $(0.6930-$ $0.7387)$, and Emergency $(<0.6930)$ by Natural Breaks methodology [33]. In Figure 19, the VSI values and states before and after DG allocation for some contingencies indicate that the operating system states are shifted to a secure position from the intermediate situation and to the intermediate state from emergency condition when DGs are placed in the system.

\section{Conclusion}

The present research employed the Symbiotic Organisms Search (SOS) algorithm for optimal Distributed Generation (DG) placement in the distribution network. Voltage security state improvement of a power network was found to be directly dependent on the bus voltage magnitude variation. This study could achieve the voltage level elevation and, consequently, voltage security enhancement in the distribution network through minimization of voltage deviation and inverse of the VSI-based multi-objective optimization problem. The results obtained from the SOS-based method were compared with those from other methodologies. The simulation findings revealed that the SOS algorithm yielded more superior results to those of Quasi-Oppositional Swine Influenza Model Based Optimization with Quarantine (QOSIMBO-Q) and Swine Influenza Based Optimization with Quarantine (SIMBO-Q). The SOS method could achieve the lowest values of the objective functions, hence superior to other previous standard techniques. The operating states of the test systems were classified into three categories based on which it can be concluded that the SOS-based DG placement could not only minimize the bus voltage magnitude deviation and increase the stability indicator value of the system, but also it elevates the voltage security level of the distribution networks under contingent conditions. As a result, the proposed methodology is used as a tool for the demand side management in the modern day.

\section{References}

1. Abri, R.S., Al, El-Saadany, E.F., and Atwa, Y.M. "Optimal placement and sizing method to improve the voltage stability margin in a distribution system using distributed generation", IEEE Transactions on Power Systems, 28(1), pp. 326-334 (2013).

2. Deb, G., Chakraborty, K., and Deb, S. "Spider monkey optimization technique-based allocation of distributed generation for demand side management", International Transactions on Electrical Energy Systems, 29(5), e12009 (2019). https://doi.org / 10.1002/20507038.12009 .

3. Tolba, M.A., Dlab, A.A.Z., Vanin, A.S., et al. "Integration of renewable distributed generation in distribution networks including a practical case study based on a hybrid PSOGSA optimization algorithm", Electric Power Components and Systems, 46, pp. 2103-2116 (2018).

4. Iqteit, N.A., Arsoy, A.B., and Çakir, B. "A timevarying load-based analytical approach for DG optimization in the distribution network", International Transactions on Electrical Energy Systems, 29(4), pp. 1-20, April (2019), https://doi.org/10.1002/etep.2783.

5. Ehsan, A. and Yang, Q. "Optimal integration and planning of renewable distributed generation in the power distribution networks: a review of analytical techniques", Applied Energy, 210, pp. 44-59 (2018). 
6. Kenari, M.T., Sepasian, M.S., and Nazar, M.S. "Probabilistic voltage stability assessment of distribution networks with wind generation using combined cumulants and maximum entropy method", International Journal of Electrical Power and Energy Systems, 95, pp. 96-107 (2018).

7. Aly, M.M. and Abdel-Akher, M. "A robust quasidirect continuous power-flow analysis using two-bus equivalents for voltage stability analysis of radial distribution systems", Electrical Engineering, 100, pp. 47-57 (2018).

8. Elsaiah, S., Benidris, M., and Mitra, J. "Analytical approach for placement and sizing of distributed generation on distribution systems", IET Generation, Transmission and Distribution, 8(6), pp. 1039-1049 (2013).

9. El-Fergany, A. "Optimal allocation of multi-type distributed generators using backtracking search optimization algorithm", International Journal of Electrical Power and Energy Systems, 64, pp. 1197-1205 (2015).

10. Ehsan, A.Q. "Optimal integration and planning of renewable distributed generation in the power distribution networks: A review of analytical techniques", Applied Energy, 210, pp. 44-59 (2018).

11. Zhang, S., Cheng, H., Li, K., et al. "Multi-objective distributed generation planning in distribution network considering correlations among uncertainties", Applied Energy, 226, pp. 743-755 (2018).

12. Sultana, U., Khairuddin, A.B., Mokhtar, A.S., et al. "Grey wolf optimizer based placement and sizing of multiple distributed generation in the distribution system", Energy, 111, pp. 525-536 (2016).

13. Othman, M.M., El-Khattam, W., Hegazy, Y.G., et al. "Optimal placement and sizing of voltage controlled distributed generators in unbalanced distribution networks using supervised firely algorithm", International Journal of Electrical Power and Energy Systems, 82, pp. 105-113 (2016).

14. Yammani, C., Maheswarapu, S., and Matam, S.K. "A multi-objective Shuffled bat algorithm for optimal placement and sizing of multi distributed generations with different load models", International Journal of Electrical Power and Energy Systems, 79, pp. 120-131 (2016).

15. Ali, E.S., Elazim, S.M.A., and Abdelaziz, A.Y. "Optimal allocation and sizing of renewable distributed generation using Ant Lion optimization algorithm", Electrical Engineering, 100, pp. 99-109 (2018).

16. Masaud, T.M., Mistry, R.D., and Sen, P.K. "Placement of large-scale utility-owned wind distributed generation based on probabilistic forecasting of line congestion", IET Renewable Power Generation, 11(7), pp. 979-986 (2017).

17. Quadri, I.A., Bhowmick, S., and Joshi, D. "A comprehensive technique for optimal allocation of distributed energy resources in radial distribution systems", $A p$ plied Energy, 211, pp. 1245-1260 (2018).
18. Abdelaziz, M. and Moradzadeh, M. "Monte-Carlo simulation based multi-objective optimum allocation of renewable distributed generation using open CL", Electric Power Systems Research, 170, pp. 81-91 (2019).

19. Zhao, Q., Wang, S., Wang, K., et al. "Multi-objective optimal allocation of distributed generations under uncertainty based on D-S evidence theory and affine arithmetic", International Journal of Electrical Power and Energy Systems, 112, pp. 70-82 (2019).

20. Da Rosa, W.M., Teixeira, J.C., and Belati, E.A. "New method for optimal allocation of distributed generation aimed at active losses reduction", Renewable Energy, 123, pp. 334-341 (2018).

21. Ud Din, F., Ahmad, A., Ullah, H., et al. "Efficient sizing and placement of distributed generators in cyber-physical power systems", Journal of Systems Architecture, 97, pp. 197-207 (2019).

22. Kumar, S., Mandal, K.K., and Chakraborty, N. "Optimal DG placement by multi-objective opposition based chaotic differential evolution for techno-economic analysis", Applied Soft Computing Journal, 78, pp. 70-83 (2019).

23. Fard, H.H. and Jalilian, A. "Optimal sizing and location of renewable energy based DG units in distribution systems considering load growth", Electrical Power and Energy Systems, 101, pp. 356-370 (2018).

24. Meena, N.K., Swarnkar, A., Gupta, N., and Niazi, K.R. "Multi-objective Taguchi approach for optimal DG integration in distribution systems", IET generation, Transmission and Distribution, 11(9), pp. 24182428 (2017).

25. Nguyen, T.P., Tran, T.T., and Vo, D.N. "Improved stochastic fractal search algorithm with chaos for optimal determination of location, size and quantity of distributed generators in distribution systems", Neural Computing and Applications, 31, pp. 77077732 (2019).

26. Gkaidatzis, P.A., Bouhouras, A.S., Doukas, D.I., et al. "Load variations impact on optimal DG placement problem concerning energy loss reduction", Electric Power Systems Research, 152, pp. 36-47 (2017).

27. Suresh, M.C.V. and Edward, J.B. "A hybrid algorithm based optimal placement of DG units for loss reduction in the distribution system", Applied Soft Computing Journal, 91, 106191 (2020).

28. Essallah, S., Khedher, A., and Bouallegue, A. "Integration of distributed generation in electrical grid: Optimal placement and sizing under different load conditions", Computers and Electrical Engineering, 79, 106461 (2019).

29. Nagaballi, S. and Kale, V.S. "Pareto optimality and game theory approach for optimal deployment of DG in radial distribution system to improve techno-economic benefits", Applied Soft Computing Journal, 92, 106234 (2020). 
30. Behera, S.G. and Panigrahi, B.K. "A multi objective approach for placement of multiple DGs in the radial distribution system", International Journal of Machine Learning and Cybernetics, 10, pp. 2027-2041 (2019).

31. Rajendran, A. and Narayanan, K. "Optimal installation of different DG types in radial distribution system considering load growth", Electric Power Components and Systems, 45(7), pp. 739-751 (2017).

32. Kansal, S., Kumar, V., and Tyagi, B. "Hybrid approach for optimal placement of multiple DGs of multiple types in distribution networks", Electrical Power and Energy Systems, 75, pp. 226-235 (2016).

33. Sharma, A., Sharma, H., Bhargava, A., et al. "Optimal placement and sizing of capacitor using Limacon inspired spider monkey optimization algorithm", Memetic Computing, 9(4), pp. 311-331 (2017).

34. Cheng, M.Y. and Prayogo, D. "Symbiotic organisms search: A new metaheuristic optimization algorithm", Computers and Structures, 139, pp. 98-112 (2014).

35. Bonab, S.M.M., Rabiee, A., Ivatloo, B.M., et al. "A two-point estimate method for uncertainty modeling in multi-objective optimal reactive power dispatch problem", International Journal of Electrical Power and Energy Systems, 75, pp. 194-204 (2016).

36. Aien, M., Hajebrahimi, A., and Firuzabad, M.F. "A comprehensive review on uncertainty modeling techniques in power system studies", Renewable and Sustainable Energy Reviews, 57, pp. 1077-1089 (2016).

37. Sharma, S., Bhattacharjee, S., and Bhattacharya, A. "Quasi-oppositional swine influenza model based optimization with quarantine for optimal allocation of DG in radial distribution network", International Journal of Electrical Power and Energy Systems, 74, pp. 348-373 (2016).

38. Chakravorty, M. and Das, D. "Voltage stability analysis of radial distribution networks", International Journal of Electrical Power and Energy Systems, 23(2), pp. 129-135 (2001).

39. Niknam, T., Taheri, S.I., Aghaei, J., et al. "A modified honey bee mating optimization algorithm for multiobjective placement of renewable energy sources", Applied Energy, 88, pp. 4817-4830 (2011).

40. Moradi, M.H. and Abedini, M. "A combination of genetic algorithm and particle swarm optimization for optimal DG location and sizing in distribution systems", International Journal of Electrical Power and Energy Systems, 34, pp. 66-74 (2012).

41. Haque, A.U., Nehrir, M.H., and Mandal, P. "A hybrid intelligent model for deterministic and quantile regression approach for probabilistic wind power forecasting", IEEE Transactions on Power Systems, 9(4), pp. 1663-1672 (2014).

42. Gong, W., Tang, H., Wang, H., et al. "Probabilistic analysis and design of stabilizing piles in slope considering stratigraphic uncertainty", Engineering Geology, 259, 105162 (2019).
43. Sharma, S., Bhattacharjee, S., and Bhattacharya, A. "Probabilistic operation cost minimization of MicroGrid", Energy, 148, pp. 1116-1139 (2018).

44. Dey, B., Bhattacharya, B., and Sharma, S. "Optimal sizing of distributed energy resources in a microgrid system with highly penetrated renewables", Iranian Journal of Science and Technology-Transactions of Electrical Engineering, 43, Supplement 1, pp. 527-540 (2019).

45. Hamada, M.M., Wahab, M.A.A, and Hemdan, N.G.A. "Simple and efficient method for steady-state voltage stability assessment of radial distribution systems", Electric Power Systems Research, 80, pp. 152-160 (2010).

46. Baz, I., Geymen, A., and Er, S.N. "Development and application of GIS-based analysis/ synthesis modeling techniques for urban planning of Istanbul metropolitan area", Advances in Engineering Software, 40, pp. 128140 (2009).

47. Ranjan, R. and Das, D. "Voltage stability analysis of radial distribution networks", Electric Power Components and Systems, 31(5), pp. 501-511 (2003).

\section{Biographies}

Kabir Chakraborty was born in Dharmanagar, Tripura, India on August 5, 1978. He is an Assistant Professor at the Department of Electrical Engineering, Tripura Institute of Technology, Narsingarh, Tripura, India. He holds a BSc in Physics (Hons.) from Assam University and BTech and MTech in Electrical Engineering from University of Calcutta. He completed his $\mathrm{PhD}$ in 2013 from Indian Institute of Engineering Science and Technology (IIEST), Shibpur, (Formerly Bengal Engineering and Science University, Shibpur,) West Bengal, India. He has 16 years of teaching and 12 years of research experience. He has published several papers in international and national journals and conference proceedings and a book.

Gagari Deb was born in Tripura on February 24, 1982. She completed her BE in Electrical Engineering from Tripura Engineering College (now NIT, Agartala) in 2004 and then, achieved her MTech in Electrical Engineering from Tripura University (A Central University) in 2008. She completed her PhD in 2021 from NIT, Agartala, India. She is currently working as an Assistant Professor at the Department of Electrical Engineering, Tripura University (A Central University). Her research area is power systems.

Sharmistha Sharma received her BE in Electrical Engineering from NIT Agartala, India (Formerly Tripura Engineering College) in 2007 and MTech in Electrical Engineering (Gold Medallist) from Tripura University (A Central University), Suryamaninagar, 
India in 2009. In 2017, she obtained her PhD degree in Power System from the Electrical Engineering Department of NIT Agartala, India. She is currently working as an Assistant Professor at the Electrical
Engineering Department of NIT Agartala, India. Her research interests include distributed generation, power and energy system, microgrid, and application of soft computing techniques to power system problems. 